\title{
Coil-to-globule transition by dissipative particle dynamics simulation
}

\author{
Jiayi Guo, ${ }^{1,2}$ Haojun Liang, ${ }^{1}$ and Zhen-Gang Wang ${ }^{2, a)}$ \\ ${ }^{1}$ CAS Key Laboratory of Soft Matter Chemistry and Hefei National Laboratory for Physical Sciences at \\ Microscale, University of Science and Technology of China, Hefei, Anhui 230026, China \\ ${ }^{2}$ Division of Chemistry and Chemical Engineering, California Institute of Technology, Pasadena, California \\ 91125, USA
}

(Received 26 January 2011; accepted 6 June 2011; published online 30 June 2011)

\begin{abstract}
The dynamics of a collapsing polymer under a temperature quench in dilute solution is investigated by dissipative particles dynamics. Hydrodynamic interactions and many-body interaction are preserved naturally by incorporating explicit solvent particles in this approach. Our simulation suggests a four-stage collapse pathway: localized clusters formation, cluster coarsening in situ, coarsening involving global backbone conformation change into a crumpled globule, and compaction of the globule. For all the quench depths and chain lengths used in our study, collapse proceeds without the chain getting trapped in a metastable "sausage" configuration, as reported in some earlier studies. We obtain the time scales for each of the first three stages, as well as its scaling with the quench depths $\xi$ and chain lengths $N$. The total collapse time scales as $\tau_{c} \sim \xi^{-0.46 \pm 0.04} N^{0.98 \pm 0.09}$, with the quench depth and degree of polymerization. (C 2011 American Institute of Physics. [doi:10.1063/1.3604812]
\end{abstract}

\section{INTRODUCTION}

The dynamics of coil-to-globule transition has been of interest for decades since the pioneering study by Stockmayer. ${ }^{1}$ Although the equilibrium properties of an isolated homopolymer under different solvent qualities are well understood, ${ }^{2}$ the dynamical behavior of a polymer when the solvent is quenched from the good solvent condition to the poor solvent condition remains controversial. Because of the strict requirements in direct experimental observation of the collapse process, ${ }^{3-5}$ such as super dilute concentration, a long polymer with polydispersity index less than 1.1, are difficult to meet, our current understanding of the transition mechanism relies primarily on theoretical and simulation studies.

The first theoretical study of the coil-to-globule transition dynamics was by de Gennes and co-workers. ${ }^{6,7} \mathrm{He}$ proposed a phenomenological model known as the "expanding sausage model." Later, Halperin and Goldbart ${ }^{8}$ proposed a "pearl-necklace model." In both models, the first stage is characterized by formation of localized blobs along the contour. Then in the "sausage model," the collapsing chain adopts a long and thin sausage conformation which gradually shortens and thickens until a crumpled globule is formed. In the "pearl-necklace model," the blobs grow in size by absorbing the beads from the bridges connecting them until the bridges become straightened; the conformation of the polymer at this stage looks like a "pearl-necklace," and finally the clusters join together and then deform into a globule. De Gennes and co-workers ${ }^{6,7}$ concluded that the collapse time $\tau_{c}$ should scale with the degree of polymerization $N$ as $\tau_{c} \sim N^{1 / 2}$. Halperin and Goldbart ${ }^{8}$ proposed three characteristic times corresponding to the three different stages, which scale as $N^{0}$, $N^{1 / 5}$, and $N^{6 / 5}$, respectively. Other phenomenological models with similar ideas were also proposed and attempts were

${ }^{a)}$ Electronic mail: zgw@cheme.caltech.edu. made on getting the scaling laws for each stage. However, there is no consensus on the exact mechanisms in each stage, and consequently there is no agreement on the scaling laws for each stage. For example, Klushin ${ }^{9}$ proposed characteristic collapse time scaling as $\tau_{c} \sim N^{0.93}$, Kikuchi et al. ${ }^{10}$ proposed $\tau_{c} \sim N^{4 / 3}\left(N^{2}\right)$ with (without) hydrodynamics. Further, Grosberg et al. ${ }^{11}$ extended de Gennes' model by taking topological constraint into consideration. According to their theory, homopolymer first collapses into an unknotted, crumpled globule and then, through a slow process that involves knotting, reaches an equilibrium globule, the characteristic times for these two steps scaling as $N^{2}, N^{3}$, respectively. Approximate theories based on Langevin dynamics ${ }^{12-18}$ similarly have not reached consensus with respect to the mechanisms and the scaling behaviors because of the different approximations invoked in the different studies. However, it is generally agreed that hydrodynamic interactions play a significant role in the transition kinetics by accelerating the collapse process.

In contrast to approximations in analytical theory and assumptions in phenomenological models, computer simulation provides a relatively straightforward way to directly capture the dynamic pathways of the transition. Because the topological knotting process is very slow, most simulation studies have focused on the formation of an unknotted crumpled globule. We similarly focus on this step during which topological constraint is not important, while other effects, such as hydrodynamic interactions, are important. Earlier work used implicit-solvent methods, such as Brownian dynamics ${ }^{19,20}$ and stochastic rotation dynamics, ${ }^{10,21,22}$ to treat the energetic and hydrodynamic interactions. The acceleration effect of hydrodynamic interactions mentioned above has been confirmed, but the collapse pathway is observed to be independent of hydrodynamic interactions, according to Pham et al. ${ }^{19}$ and Kikuchi and co-workers. ${ }^{10,21}$ Their results, respectively, showed that when the quench depth is low, polymer collapse follows the "pearl-necklace" model into a globule smoothly; 
while when the quench depth is large, the pearl-necklace stage is rapid and a fat and short sausage forms. In the latter case, the polymer is observed to be frequently trapped as a sausage and cannot deform into a globule irrespective of whether hydrodynamic interactions are included. Clearly, the sausage conformation is a metastable state. Chang and Yethiraj $^{23}$ and Polson and Zuckermann ${ }^{24,25}$ used molecular dynamics (MD) with explicit solvent beads and found the polymer collapses smoothly without getting trapped, for all the different quench depths and different chain lengths they studied. They concluded that collision by the solvent particles can prevent trapping. Later, Reddy and Yethiraj ${ }^{26}$ used a new simulation method which does not include hydrodynamic interactions but mimics the many-body interactions by taking into account the solvent accessible surface area. They found that all their samples collapse into the equilibrium globule without getting trapped. Tanaka and co-workers ${ }^{27}$ used another method, known as fluid particle dynamics, which treats the solvent implicitly and can mimic many-body hydrodynamic interactions down to short length scales. Their simulation showed that hydrodynamic interactions accelerate collapse from a swollen chain, but retard collapse from a slightly crumpled coil. They concluded that the many-body hydrodynamic interactions delay the polymer beads from getting into direct contact because of "squeezing out" effect and thus prevent trapping. However, their simulation showed the collapse follows "sausage" mechanism which is different from other simulations and from our results to be reported here, and the work also did not obtain scaling exponents. It is clear from these studies that the many-body solvent-induced interactions, of both energetic and hydrodynamic nature, are important in order to provide a reliable description of the kinetics of polymer collapse. We also note that none of these simulations studies have reached a consensus on the scaling laws for the various dynamic properties.

It should be clear from the preceding discussions that an appropriate simulation approach must account for the hydrodynamic interactions, treat solvent particles explicitly, and is also computationally efficient. The dissipative particle dynamics (DPD) is a mesoscopic method that fulfills all these requirements. While the MD method can naturally account for the solvent particles and automatically includes hydrodynamics, the mesoscopic nature of the DPD method allows us to reach longer times, using larger simulation boxes with longer chains. In this work, we perform a DPD study of the kinetics of the coil-to-globule transition and focus on the formation of the unknotted globule. With the computational advantages afforded by the DPD method, we hope that our study will provide more definitive results on the transition pathways and the scaling behavior of the dynamic observables.

Generally, we find that when quench depth is not too deep the collapse follows the "pearl-necklace" mechanism and there are four kinetic stages: (1) a rapid, localized cluster formation; (2) a coarsening stage in situ; (3) a coarsening stage involving global backbone conformation changing into a crumpled globule; and (4) compaction of the globule. The last stage will not be discussed here, as it most likely will involve knotting ${ }^{11}$ and will require very long simulation times. When the quench depth is very deep, each of the first three stage is rapid and the distinction between the different stages becomes less clear: the pearl-necklace quickly condenses into a short and fat ellipsoid and the ellipsoid then is observed to deform into a globule. No trapping phenomenon is observed in either case. We investigate the scaling laws of the durations of the first three stages with different quench depths and different chain lengths. The total collapse time $\tau_{c}$ scales with the quench depth $\xi$ (defined as the difference in the interaction parameter from its value at the coil-globule transition) and degree of polymerization $N$ as $\tau_{c} \sim \xi^{-0.46 \pm 0.04} N^{0.98 \pm 0.09}$.

\section{METHOD AND MODEL}

\section{A. Dissipative particle dynamics}

The DPD was developed by Hoogerbrugge and Koelman $^{28,29}$ in 1992. In this method, each bead represents several molecules or molecular groups. For convenience, we assume the sizes and masses of all beads are the same. The cutoff radius $r_{c}$ of bead for interactions is set to be 1 , and the mass of each bead is also set to be 1 . The velocity and position of each bead follow Newton's equations:

$$
\frac{d \mathbf{r}_{i}}{d t}=\mathbf{v}_{i}, \frac{d \mathbf{v}_{i}}{d t}=\mathbf{f}_{i},
$$

in which $\mathbf{r}_{i}, \mathbf{v}_{i}$, and $\mathbf{f}_{i}$ are the position, velocity, and force of bead $i$, respectively. The force on each bead contains three pairwise additive parts: a conservative force, $\mathbf{F}_{i j}^{C}$; a dissipative force, $\mathbf{F}_{i j}^{D}$; and a random force, $\mathbf{F}_{i j}^{R}$,

$$
\mathbf{f}_{i}=\sum_{j \neq i}\left(\mathbf{F}_{i j}^{C}+\mathbf{F}_{i j}^{D}+\mathbf{F}_{i j}^{R}\right),
$$

where the force $\mathbf{f}_{i}$ include contributions from all the beads within the cutoff radius $r_{c}$. Each term in Eq. (2) is defined as

$$
\begin{aligned}
& \mathbf{F}_{i j}^{C}=\left\{\begin{array}{ll}
a_{i j}\left(1-r_{i j} / r_{c}\right) \widehat{\mathbf{r}}_{i j}, & r_{i j} \leq r_{c} \\
0, & r_{i j}>r_{c}
\end{array},\right. \\
& \mathbf{F}_{i j}^{D}=-\gamma \omega^{D}\left(r_{i j}\right)\left(\widehat{\mathbf{r}}_{i j} \cdot \mathbf{v}_{i j}\right) \widehat{\mathbf{r}}_{i j}, \\
& \mathbf{F}_{i j}^{R}=\sigma \zeta_{i j} \omega^{R}\left(r_{i j}\right) \widehat{\mathbf{r}}_{i j},
\end{aligned}
$$

where $a_{i j}$ is the maximum repulsion between beads $i$ and $j$, and $\mathbf{r}_{i j}=\mathbf{r}_{j}-\mathbf{r}_{i}, r_{i j}=\left|\mathbf{r}_{i j}\right|, \widehat{\mathbf{r}}_{i j}=\mathbf{r}_{i j} / r_{i j}, \gamma$ is a viscosity coefficient, and $\sigma^{2}=2 \gamma k_{B} T . \mathbf{v}_{i j}=\mathbf{v}_{j}-\mathbf{v}_{i}$ is the relative velocity between beads $i$ and $j$. Here, $\omega^{D}$ and $\omega^{R}$ are $r$-dependent weight functions taken to be of the form,

$$
\omega^{D}=\left[\omega^{R}\right]^{2}=\left\{\begin{array}{ll}
\left(1-r_{i j} / r_{c}\right)^{2}, & r_{i j} \leq r_{c} \\
0, & r_{i j}>r_{c}
\end{array},\right.
$$

where $\zeta_{i j}$ is a random fluctuating variable following Gaussian statistics,

$$
\left\langle\zeta_{i j}(t)\right\rangle=0,\left\langle\zeta_{i j}(t) \zeta_{k l}\left(t^{\prime}\right)\right\rangle=\left(\delta_{i k} \delta_{j l}+\delta_{i l} \delta_{j k}\right) \delta\left(t-t^{\prime}\right) .
$$

In the present simulation, we connect consecutive particles with a spring force to construct a full flexible polymer chain. ${ }^{30}$ The Fraenkel spring constant of the bond is $K_{S}$ 
$=80.0 k_{B} T / r_{c}^{2}$ with the equilibrium spring length $r_{e q}$ $=0.86 r_{c}$ and the form is $F_{i j}^{S}=K_{S}\left(r_{i j}-r_{e q}\right) \mathbf{e}_{i j}$. Other parameters used here are $k_{B} T=1.0$ which is the energy scale and a dissipation coefficient $\sigma=3.0\left(\varepsilon^{3} m / r_{c}^{2}\right)^{1 / 3}$.

The present simulation contains two types of particles representing polymer $P$ and solvent $S$. The interaction parameters between the three types of pairs are $a_{P P}=a_{S S}=$ $25.0 k_{B} T / r_{c}$, and $a_{P S}$ is varied. The larger this interaction parameter is, the more repulsive is between a polymer segment and a solvent particle, which corresponds to a deeper quench.

Our simulations are performed on cubic boxes with periodic boundaries along the three directions. In order to eliminate the effect of the finite box size, we consider different box sizes corresponding to different polymer chain lengths, such as $L=30.0 r_{c}$ with 80 polymer beads, $L=40.0 r_{c}$ with 120 and 160 polymer beads, $L=50.0 r_{c}$ with 240 and 320 polymer beads. The equation of motion is integrated using modified velocity-Verlet algorithm with $\lambda=0.65$ and time step $\Delta t=0.02 \tau$, where the DPD time scale $\tau=\left(m r_{c}^{2} / k_{B} T\right)^{1 / 2}$.

\section{B. Units}

In this paper, we use scaled length unit $r_{c}$ and time unit $\tau$. To map from the scaled units to physical units, we follow Groot and Rabone's method ${ }^{31}$ and estimate the length and time scales as follows.

We take the solvent molecule to be of the same size as water. The volume of a water molecule is $30 \AA^{3}$ and each solvent bead in DPD contains three water molecules, and each cell of length $r_{c}$ of DPD contains three solvent beads. Therefore, $r_{c}=\sqrt[3]{270} \AA=6.4633 \AA$.

Then assume $R_{0}$ is the center of mass of the solvent bead which contains three real water molecules corresponding to $R_{1}, R_{2}$, and $R_{3}$. The mean-square displacement of solvent bead is $R_{0}^{2}=\left(\left\langle R_{1} \cdot R_{1}\right\rangle+\left\langle R_{2} \cdot R_{2}\right\rangle+\left\langle R_{3} \cdot R_{3}\right\rangle\right) / 9=R^{2} / 3$, in which $R^{2}$ is the mean square displacement of a real water molecule. We took average among 10000 samples and obtained the diffusion constant of the water bead according to $\left\langle R_{0}(t)-R_{0}(0)\right\rangle^{2}=6 D t$ and eventually got $D=0.29 r_{c}^{2} / \tau$. The real water diffusion constant is $2.43 \times 10^{-5} \mathrm{~cm}^{2} / \mathrm{s}$. Thus, $D=\frac{2.43 \times 10^{-5} \mathrm{~cm}^{2} / \mathrm{s}}{3}$ and we get $\tau \approx 1.5 \times 10^{-10} \mathrm{~s}=1.5 \mathrm{~ns}$.

\section{Definition of a cluster}

In this paper, we record the growth of the average cluster size with time by the number-average $\left\langle S_{n}(t)\right\rangle$. The definition is as follows:

$$
\left\langle S_{n}(t)\right\rangle=\frac{\sum_{s} s n(s)}{\sum_{s} n(s)},
$$

in which $s$ is the cluster size, i.e., the number of beads in the cluster; $n(s)$ denotes the number of clusters of size $s$. We follow the same way as other authors to define a cluster, ${ }^{15,19}$ i.e., non-bonded beads are considered to be in the same cluster when the separation between each pair of them is less than the cutoff radius $r_{c}$.

\section{RESULTS AND DISCUSSION}

\section{A. Locating the $\theta$-point}

The " $\theta$-point" of a polymer chain is the condition where the excluded volume interaction is exactly balanced by the effective attraction between the monomers, so that the chain behaves ideal. Thermodynamically this condition corresponds to the vanishing of the second virial coefficient for monomermonomer interactions. The mean square radius of gyration of a polymer in different solvent conditions should follow: ${ }^{2}$

$$
\left\langle R_{g}^{2}\right\rangle / N \sim \begin{cases}N^{1 / 5}, & \text { good } \\ 1, & \theta \\ N^{-1 / 3}, & \text { poor. }\end{cases}
$$

Because at the $\theta$-point $\left\langle R_{g}^{2}\right\rangle / N$ is independent of chain lengths, we can locate the $\theta$-point by plotting this quantity as a function of the interaction parameter for different chain lengths and finding the point at which the different curves meet; ${ }^{32,33}$ see Fig. 1 . With this method, we obtain the value of the interaction parameter at the $\theta$-point to be $a_{P S}^{\theta} \approx 27.0 k_{B} T / r_{c}$.

Because the transition from good solvent to poor solvent across the $\theta$-point is not sharp for polymers of finite chain lengths, below the theta temperature or above $a_{P S}^{\theta}$, there is still a range in which the polymer retains its behavior as a coil. $^{32,34}$ Therefore, we choose $a_{P S}$ large enough to ensure that the final equilibrium conformation is a compact globule.

\section{B. Kinetics of the transition}

Our simulation starts with polymer chains which have been equilibrated in good (athermal) solvent conditions, i.e., $a_{P P}=a_{S S}=a_{P S}=25.0 k_{B} T / r_{c}$ for durations longer than their relaxation time. Then the systems are quenched into poor solvent conditions by abruptly increasing the interaction parameter $a_{P S}$ above the transition point. In Fig. 2, the snapshots capture the typical collapse pathway of a polymer chain with $N=320$ beads at repulsive parameter

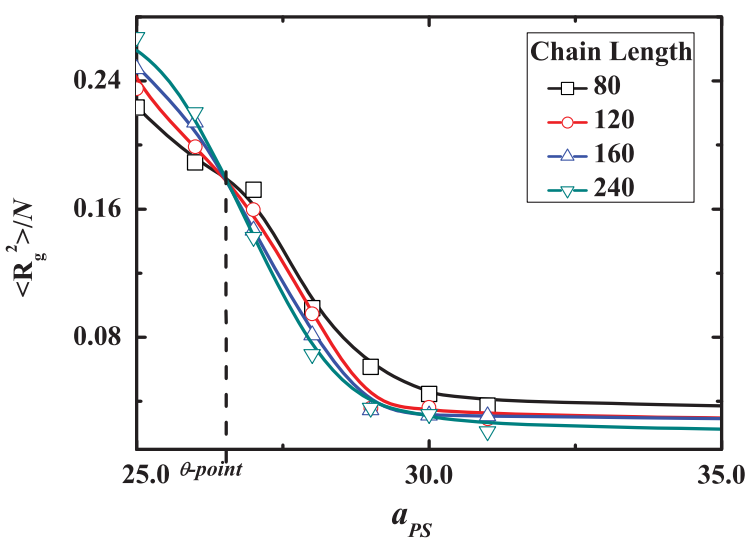

FIG. 1. Scaled mean square radius of gyration $\left\langle R_{g}^{2}\right\rangle / N$ as a function of interaction parameter for four different chain lengths. The $\theta$-point is located at $a_{P S}^{\theta} \approx 27.0 k_{B} T / r_{c}$. 

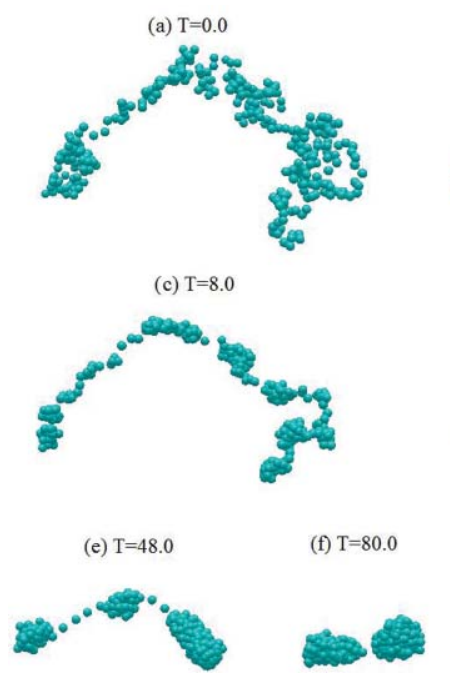

(g) $\mathrm{T}=90.0$

(h) $\mathrm{T}=100.0$

FIG. 2. Pathway of a collapsing polymer with 320 beads at $a_{P S}$ $=60.0 k_{B} T / r_{c}$.

$a_{P S}=60.0 k_{B} T / r_{c}$. The time shown is in dimensionless DPD units. Here, $t=0$ corresponds to the time when the system is suddenly quenched from the athermal solvent condition into the poor solvent condition.

We have performed simulations with different quench depths and chain lengths. In all the simulations, the collapse follows a pearl-necklace pathway. Figure 2 shows a typical sequence of events along the transition pathway. According to the snapshots, three different stages can be identified: rapid formation of local clusters (Fig. 2(b)), in situ coarsening (Fig. 2(c)), coarsening involving global backbone conformation change into a globule (Figs. 2(c)-2(h)). As an aggregate measure of the collapsing process, we monitor the evolution of the mean-square radius of gyration with time; this is shown in Fig. 3. The curves are all seen to be monotonic and smooth, with no obvious pause, which means that there is no trapping in our system. It can also be seen that the deeper the quench depth is, the faster the collapse is.

During collapse, the polymer beads cluster to minimize contact with solvent particles. The average number of solvent particles in contact with a polymer bead $\left\langle N_{S}\right\rangle$ vs. $t$ is recorded in Fig. 4. The time-dependent $\left\langle N_{S}(t)\right\rangle$ can be normalized as $\bar{N}_{S}(t)=\left(\left\langle N_{S}(t)\right\rangle-N_{\infty}\right) /\left(\left\langle N_{S}(0)\right\rangle-N_{\infty}\right)$ and three stages of the collapse pathway can be found corresponding to each stage mentioned above (Figs. 4(a) and 4(b)). During the first stage, $\bar{N}_{S}(t)$ decrease rapidly, and then the rest of the curve can be fitted approximately by two different exponential functions. By this way, we investigate the effect of different quench depths and chain lengths on the duration of each stage during collapse. We now describe each stage separately in some detail.

Stage 1: The first stage is characterized by rapid formation of localized clusters along the polymer chain after a sudden quench (see Figs. 2(a) and 2(b)). The locations of localized clusters after quench are regions which possess a relatively high density of beads along the chain due to fluctuation before the quench (Fig. 2(a)). Initially, the polymer chain undergoes local contraction as the clusters start to form along
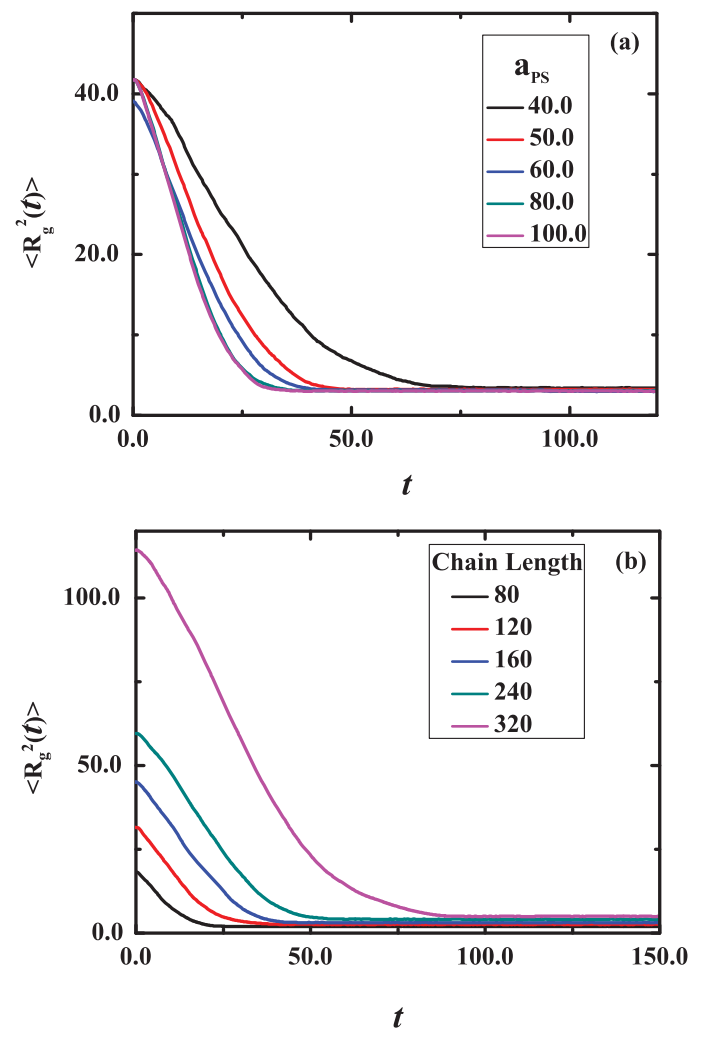

FIG. 3. (a) $\left\langle R_{g}^{2}(t)\right\rangle$ vs. $t$ for different quench depths; the polymer length is $N=160$ beads. (b) $\left\langle R_{g}^{2}(t)\right\rangle$ vs. $t$ for different chain lengths. The repulsive parameter is $a_{P S}=60.0 k_{B} T / r_{c}$. Each curve in Fig. 3 is averaged over 20 samples for polymers with $80-160$ beads, and 16 samples for polymers with 240 and 320 beads, respectively.

the chain. Solvent particles are squeezed out during this process. The dots on each curve set the end of this process as shown in the insets of Figs. 4(a) and 4(b). It is clear that the deeper the quench depth is, the faster the process. We find that this stage is well fitted with Gaussian function, i.e., $\bar{N}_{S}(t)$ $=\exp \left(-t^{2} /\left(2 \tau_{1}^{2}\right)\right)$, we obtain approximately $\tau_{1} \sim \xi^{-0.36 \pm 0.01}$ for polymer chain length $N=160$ as shown in Table I, in which $\tau_{1}$ is the time scale of the local contract process. Similarly, we get other scaling exponents as $-0.36 \pm 0.01$, $-0.40 \pm 0.06,-0.38 \pm 0.07$, and $-0.43 \pm 0.07$ for chain length $N=80,120,240$, and 320 , respectively. Because this process occurs on a local scale, we do not expect any chain length dependence; indeed, we find no chain length dependence in $\tau_{1}$ (Table II shows $\tau_{1}$ for different chain lengths at a fixed quench depth $a_{P S}=60.0 k_{B} T / r_{c}$ ). Generally, we find the scaling law of this stage as $\tau_{1} \sim \xi^{-0.39 \pm 0.05} N^{0}$. Independence of this time scale on the chain length has also been obtained by other authors, ${ }^{8,14}$ while no previous result has been given on the scaling with the quench depths $\xi$.

After the rapid local contraction, polymer beads begin to gather to form clusters. Interestingly the process is not a simple monotonic one, but involves a visible overshoot and small oscillations; see insets in Figs. 4(a) and 4(b). We do not have a simple explanation for this phenomenon, except to note that the local contraction may lead to a rapid increase of tension along the chain backbone, which must subsequently relax. Several researchers $8,13,14$ argued that the first stage is 

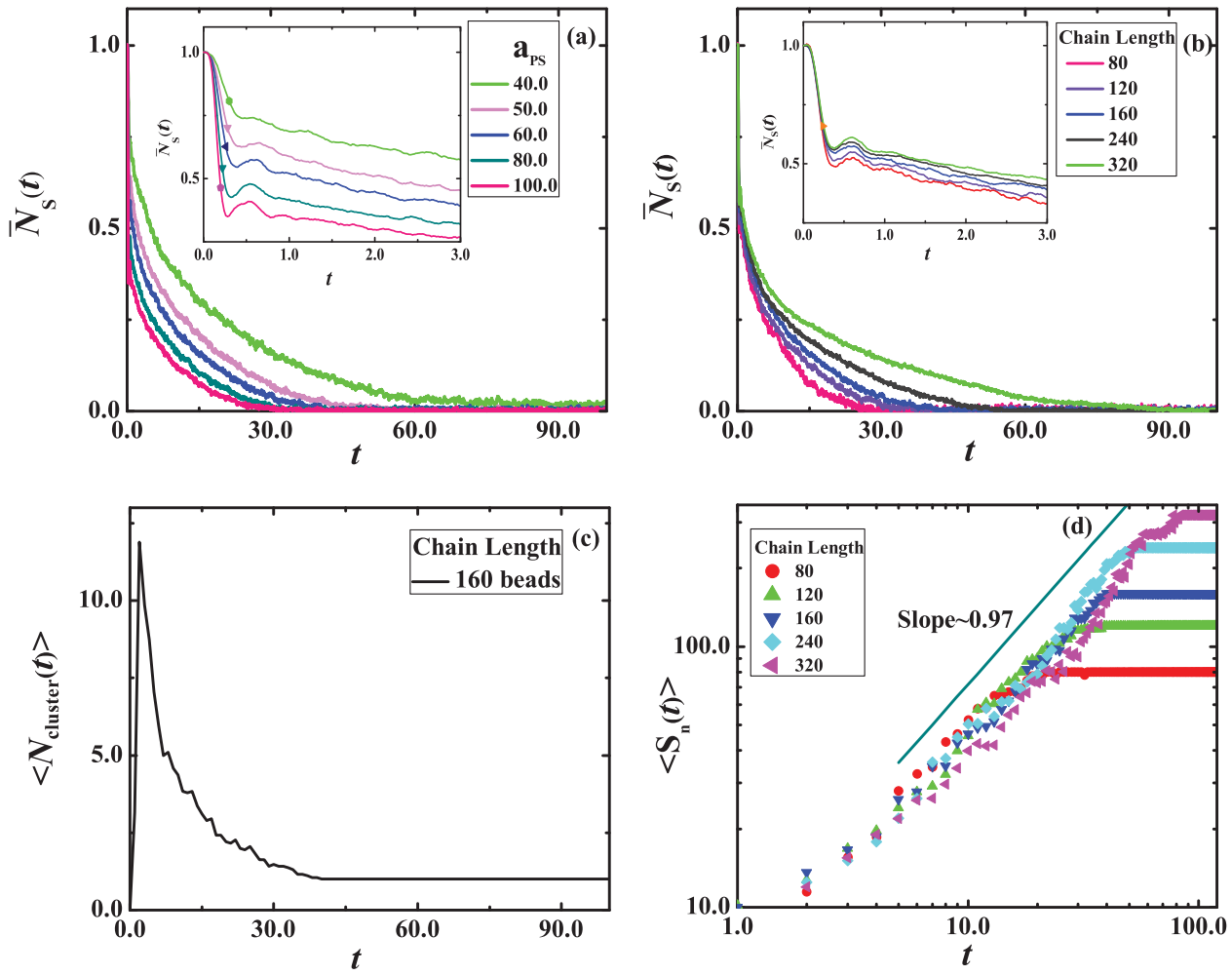

FIG. 4. (a) Normalized average number of solvent particles in contact with a polymer bead $\bar{N}_{S}(t)$ vs. $t$ during collapse for the same chain length $N=160$ beads at different quench depths. (b) $\bar{N}_{S}(t)$ vs. $t$ during collapse for the different chain lengths at the same repulsive parameter. The insets in both (a) and (b) show the first stage, with the dots indicating the time scale of this stage. (c) Average number of clusters along the contour $\left\langle N_{\text {cluster }}(t)\right\rangle$ vs. $t$ for the polymer with 160 beads at $a_{P S}=60.0 k_{B} T / r_{c}$. (d) A log-log plot of the number average cluster size $\left\langle S_{n}(t)\right\rangle$ as a function of time $t$ during collapse of different chain lengths at $a_{P S}=60.0 k_{B} T / r_{c}$. The straight line is fitted to the linear region of the curves.

akin to "spinodal decomposition," and some identified a characteristic blob size. Visual inspection of the collapse transition pathway indeed shows resemblance to spinodal decomposition; however, it is difficult to quantify the characteristic blob size and "uphill" diffusion against the density gradient ${ }^{35}$ in the current simulation.

Following the first stage is the coarsening process during which clusters absorb nearby beads and coalescence with other clusters. As a result, clusters grow while their number decreases (Figs. 4(c) and 4(d)). We separate it into two stages by fitting into two exponentials, using the physical picture of the collapse pathway as a guide. For the stage 2 which immediately follows the first stage, we smooth out the oscillations using a standard numerical algorithm — adjacent averaging. We characterize the time scale for each stage and note little similarity between our results and results from previ- ous works. Generally, the time scales are longer if the quench depth is shallower and/or the chain is longer.

Stage 2: This stage corresponds to the coarsening of the clusters in situ (Fig. 2(c)). At the beginning of this stage, clusters are small, typically only containing $3-5$ beads, and they grow in size mainly by absorbing polymer beads and some other small clusters that form the bridges between clusters. Then larger clusters form and roughly stay stationary while single beads and smaller clusters approach them as single beads and smaller clusters in the bridges that are not taut have higher mobility. Gradually, the "wiggly" bridges are straightened. This is also a fairly rapid process but much slower than the first stage. We measure the time scale of this process from the intersection between the fitting for this stage and that for the following stage and find $\tau_{2} \sim \xi^{-0.32 \pm 0.02}$ for the chain length $N=160$ as shown in Table I. For other

TABLE I. Durations for each stage at different quench depths for the same chain length of 160 beads. $\gamma$ is the apparent exponent in the scaling of the time scale of each stage with the quench depth (i.e., $\tau \sim \xi^{\gamma}$ ).

\begin{tabular}{lccccc}
\hline \hline$a_{P S}$ & $\xi$ & $\tau_{1}$ & $\tau_{2}$ & $\tau_{3}$ & $\tau_{c}$ \\
\hline 40.0 & 0.48 & 0.15 & 7.65 & 61.86 & 69.78 \\
50.0 & 0.85 & 0.12 & 6.99 & 41.52 & 48.63 \\
60.0 & 1.22 & 0.11 & 5.77 & 36.01 & 41.89 \\
80.0 & 1.96 & 0.09 & 5.01 & 31.32 & 36.42 \\
100.0 & 2.70 & 0.08 & 4.58 & 26.03 & 30.69 \\
$\gamma$ & & $-0.36 \pm 0.01$ & $-0.32 \pm 0.02$ & $-0.47 \pm 0.05$ & $-0.45 \pm 0.04$ \\
\hline
\end{tabular}


TABLE II. Durations for each stage of different chain lengths at the same repulsive parameter $a_{P S}=60.0 k_{B} T / r_{c} . \gamma$ is the exponent in the apparent scaling of the time scale of each stage with the chain length (i.e., $\tau \sim N^{\gamma}$ ).

\begin{tabular}{lcccc}
\hline \hline Chain length $N$ & $\tau_{1}$ & $\tau_{2}$ & $\tau_{3}$ & $\tau_{c}$ \\
\hline 80 & 0.11 & 4.90 & 19.38 & 24.39 \\
120 & 0.12 & 5.98 & 27.74 & 33.84 \\
160 & 0.11 & 5.77 & 36.01 & 41.89 \\
240 & 0.11 & 11.43 & 50.47 & 62.01 \\
320 & 0.12 & 13.09 & 72.40 & 85.61 \\
$\gamma$ & 0 & $*^{\mathrm{a}}$ & $0.93 \pm 0.04$ & $0.90 \pm 0.03$ \\
\hline \hline
\end{tabular}

${ }^{a} *$ indicates that the quality of the data is not good enough to obtain a meaningful scaling exponent.

chain lengths $N=80,120,240$, and 320 , the scaling exponents are $-0.43 \pm 0.10,-0.25 \pm 0.02,-0.23 \pm 0.03$, and $-0.23 \pm 0.03$, respectively. Halperin and Goldbart ${ }^{8}$ proposed $\tau_{2} \sim N^{1 / 5}$ for this stage, but the quality of our data (Table II) is not sufficient to allow us to obtain an exponent with reasonable accuracy. It is clear, however, that the time scale increases with the chain length. Combining the results for different chain lengths, we obtain the scaling law of this stage as $\tau_{2} \sim \xi^{-0.30 \pm 0.05}$.

Stage 3: As the bridges are straightened, clusters grow in size mainly by moving in the solvent and coalescing with each other (Figs. 2(c)-2(h)). The decreased mobility of clusters (as a consequence of their increased size) results in a slower decrease of $\bar{N}_{S}(t)$ and longer duration compared to the second stage. According to the thesis of some earlier works, at this stage, clusters are regarded as a spherical ball and there exist an energy barrier in bringing two different clusters close. ${ }^{8,9}$ But in our simulations, we find that clusters undergo facile shape distortion when approaching each other (see discussions below) and coalescence rather quickly, without any apparent barrier.

Shape distortion is allowed by the fluctuation of cluster beads in their position. During this stage, clusters are not yet quite compact and are relatively small; a large fraction of the beads, especially those on the surface are in contact with the solvent molecules. These beads are subjected to frequent collision with solvent particles, and hence the polymer beads are subjected to large fluctuations in their position. A quantitative measure of the fluctuations of these beads can be obtained by examining the mean-square amplitudes of the normal modes of the polymer. In Fig. 5, we plot the amplitude of the $p$ modes $\left\langle X_{p}^{2}(t)\right\rangle$ of the polymer. The small $p$-modes $\left\langle X_{p}^{2}(t)\right\rangle$ represent relatively global fluctuation of polymer and large ones represent relatively local fluctuation of polymer. The amplitude of the $p$-modes are observed to decrease toward equilibrium, implying local structures at such length gradually are included into clusters of increasing size. As shown in Fig. 5(a), $\left\langle X_{p}^{2}(t)\right\rangle$ reach equilibrium from $p=8$ to $p=1$ one by one as clusters grow larger and larger. But as shown in Fig. 5(b), the curves of $\left\langle X_{80}^{2}(t)\right\rangle$ and $\left\langle X_{160}^{2}(t)\right\rangle$, which represent single monomer fluctuation, do not reach equilibrium until the end of the collapse. We note that in Ref. 14, large $p$-modes fall to equilibrium rapidly, which is quite different
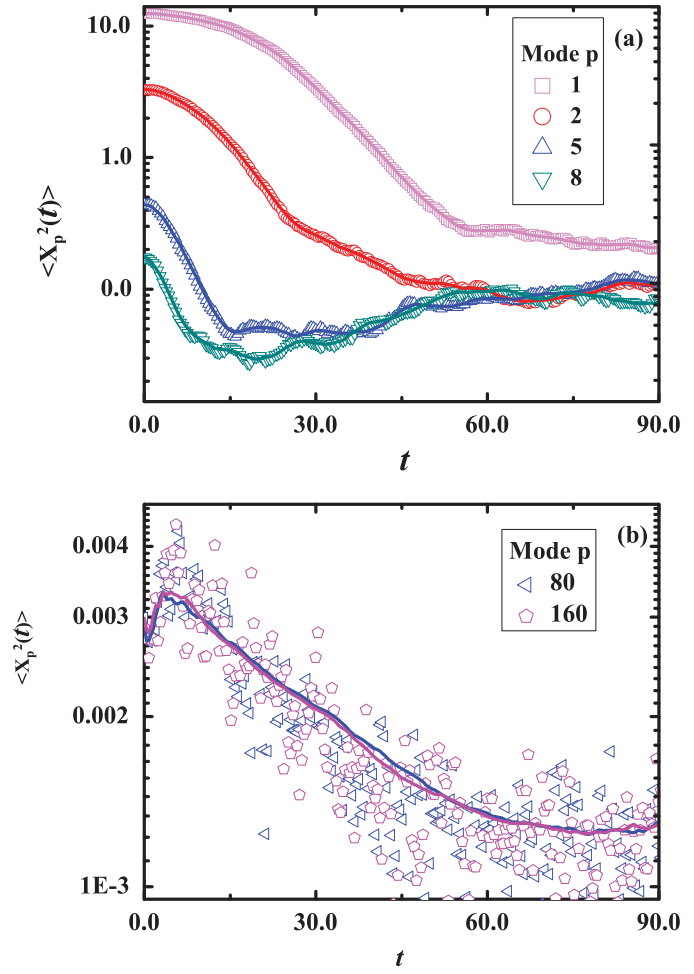

FIG. 5. (a) Amplitude of normal mode $\left\langle X_{p}^{2}(t)\right\rangle$ vs. time step $t$ of a polymer with 160 beads at repulsive parameter $a_{P S}=60.0 k_{B} T / r_{c}$ in which $p=1,2$, $5,8,80$, and 160, respectively. $\left\langle X_{80}^{2}(t)\right\rangle$ and $\left\langle X_{160}^{2}(t)\right\rangle$ keep decreasing until equilibrium at the end of the transition. The lines are used to guide readers.

from ours. This difference could be due to both their use of the implicit solvent model and the introduction of approximations in their analysis.

When some of clusters get close to each other, although clusters have lower mobility due to their size and the constraint of the polymer chain, the outer polymer beads with high fluctuation in their position allows clusters to undergo shape distortion that can help two separated clusters get into contact and then join together. The clusters that just touch soon merge into a new bigger spherical one because of capillary forces as sausage-like clusters are unstable in solvent, which is also discussed by other works. ${ }^{8,9,36}$ This stage follows a pearl-necklace mechanism with decreasing number of "pearls" along the "necklace." In almost all of the samples, we find that the "pearl-necklace" shortens to a dumbbell conformation at the late coarsening stages (Fig. 2(f)). We reason that the dumbbell conformation is a consequence of the higher mobility of two end-clusters which retract from the ends and grow and dominate by coalescing with the neighboring clusters. This phenomenon was discussed in some detail in the work by Ostrovsky et al. . $^{37,38}$

Eventually, the dumbbell joins together into an ellipsoid (Figs. 2(f) and 2(g)). As the new sausage-like cluster is not yet compact, the collision with solvent particles provides the mechanism for it to search for a lower-energy state and thus the sausage-shaped cluster deforms into a crumpled globule smoothly (Figs. 2(g) and 2(h)). In contrast, in methods which treat the solvent implicitly, the solvent-monomer repulsion 
TABLE III. Overall collapse time with different quench depths and different chain lengths. $\gamma_{1}$ is the exponent in the apparent scaling of the time scale with the quench depths (i.e., $\tau \sim \xi^{\gamma_{1}}$ ). $\gamma_{2}$ is the exponent in the apparent scaling of the time scale with the quench depths (i.e., $\tau \sim \xi^{\gamma / 2}$ ).

\begin{tabular}{lcccccc}
\hline \hline$\xi$ & $\tau_{80}$ & $\tau_{120}$ & $\tau_{160}$ & $\tau_{240}$ & $\tau_{320}$ & $\gamma_{2}$ \\
\hline 0.48 & 37.12 & 51.69 & 69.78 & 96.73 & 163.05 & $1.03 \pm 0.09$ \\
0.85 & 27.83 & 38.30 & 48.63 & 63.85 & 121.49 & $0.99 \pm 0.14$ \\
1.22 & 24.39 & 33.84 & 41.89 & 62.01 & 85.61 & $0.90 \pm 0.03$ \\
1.96 & 19.65 & 27.22 & 36.42 & 47.24 & 81.08 & $0.95 \pm 0.09$ \\
2.70 & 16.82 & 23.07 & 30.69 & 45.55 & 70.43 & $1.02 \pm 0.07$ \\
$\gamma_{1}$ & $-0.45 \pm 0.01$ & $-0.45 \pm 0.02$ & $-0.45 \pm 0.04$ & $-0.44 \pm 0.06$ & $-0.49 \pm 0.06$ & \\
\hline \hline
\end{tabular}

is represented by monomer-monomer attraction. When the quench depth is low, the attraction between monomers can be readily overcome by thermal fluctuation and the sausagelike cluster can search for the lower-energy state and reach equilibrium into a spherical one without being trapped. But when the quench depth is deep, the attraction between polymer beads can be so strong that the relatively small thermal fluctuation is insufficient to help the sausage deform into a globule on short time scales. This is probably the reason that, in implicit solvent models, the conformation of the polymer is frequently trapped in the sausage-like metastable state. ${ }^{10,19,27,39}$ The duration of this stage roughly scales as $\tau_{3} \sim \xi^{-0.47 \pm 0.05}$ for $N=160$ and $\tau_{3} \sim N^{0.93 \pm 0.04}$ at $a_{P S}$ $=60.0 k_{B} T / r_{c}$ as shown in Tables I and II. We also obtain the scaling exponents as $-0.45 \pm 0.03,-0.50$ $\pm 0.02,-0.47 \pm 0.07$, and $-0.56 \pm 0.08$ for the chain length $N=80,120,240$, and 320 , respectively; and $1.06 \pm 0.10,1.03 \pm 0.16,0.95 \pm 0.13$, and $0.97 \pm 0.07$ for $a_{P S}=40.0,50.0,80.0,100.0 k_{B} T / r_{c}$, respectively. Thus the overall scaling law of this stage is approximately $\tau_{3}$ $\sim \xi^{-0.49 \pm 0.05} N^{0.99 \pm 0.11}$. Halperin and Goldbart ${ }^{8}$ once got $\tau_{3}$ $\sim N^{6 / 5}$ which has a larger exponent than our results.

As a result of the coarsening process including second and third stages, the number of clusters decreases and their sizes increase. During this process, the growth of the average cluster size $\left\langle S_{n}(t)\right\rangle$ with time $t$ at a given quench $a_{P S}$ $=60.0 k_{B} T / r_{c}$ is recorded as shown in Fig. 4(d). The log$\log$ plots show power-law behavior for the growth of the average cluster size with time as $\langle S\rangle=A t^{z}$, where $z$ is a collapse exponent. We obtain scaling exponents $z$ as $0.98 \pm 0.11$, $0.95 \pm 0.07,0.93 \pm 0.11,0.97 \pm 0.04$, and $0.94 \pm 0.07$ and repulsive parameters $a_{P S}=40.0,50.0,60.0,80.0$, and 100.0, respectively. Thus during the coarsening process, $\left\langle S_{n}(t)\right\rangle$ $\sim t^{0.95 \pm 0.08}$. We note that similar results were also obtained in the work of Pham et al., ${ }^{19}$ but not in other previous works. We also note that the growth exponents for $\left\langle S_{n}(t)\right\rangle$ is larger than Klushin's ${ }^{9}$ and Byrne et al.'s ${ }^{15}$ theoretical predictions. We suspect that the cluster shape fluctuation that was not considered in these theoretical studies can accelerate the coalescence of the clusters.

We note that numerically the curves in Figs. 4(a) and 4(b) during stage 3 can be better fitted by two different exponential functions, with two different relaxation times. This would suggest that stage 3 can be further divided into two separated stages. Visual inspection of the collapse pathway seems to show that the first part corresponds to coarsening associated with backbone conformation change until the coalescence of the "dumbbell," while the latter part corresponds to rounding of an ellipsoidal shaped globule into a spherical one. However, the two time scales associated with these two processes are very similar with respect to their scaling in the quench depth and chain length. Given the error bars in the simulation, we are unable to definitively state whether the two processes constitute different mechanistic stages.

Stage 4: This is the relaxation towards the final equilibrium globule. Experimentally, this stage has been examined by several groups. ${ }^{3-5}$ But it is difficult to find this stage by monitoring $\left\langle R_{g}^{2}(t)\right\rangle$ and $\left\langle N_{S}(t)\right\rangle$ in our simulation as these two dynamic measures are insensitive to the small (largely internal) rearrangements. However, analysis of the normal modes as shown in Fig. 5(b) reveals that the large- $p$ modes continue to decrease long after $\left\langle R_{g}^{2}(t)\right\rangle$ and $\bar{N}_{S}(t)$ reached their equilibrium values. As discussed by Grosberg et al., ${ }^{11}$ for long polymers, this stage most likely involves the selfentanglement due to knotting, which will require very long simulation times. Furthermore, the softness in the potential used in the DPD method is not strong enough to prevent chain crossing. Therefore, we will not discuss this stage further in this paper.

Operationally, the characteristic duration of the collapse transition $\tau_{c}$ can be calculated as $R_{g}^{2}(t)=(1 / 100)\left(R_{g}^{2}(0)\right.$ $\left.\left.-\left\langle R_{g}^{2}\right\rangle_{e q}\right)+\left\langle R_{g}^{2}\right\rangle_{e q}\right) .{ }^{14,19}$ During the last stage, the change in $\left\langle R_{g}^{2}(t)\right\rangle$ is insignificant, so the characteristic duration of the transition defined this way primarily involves the first three stages. Here we add the duration of each stage as a measure of the collapse time; the behaviors of these two differently defined collapse times are similar. The results in Table III show the scaling of the characteristic collapse time with the quench depth $\xi=\left(a_{P S}-a_{P S}^{\theta}\right) / a_{P S}^{\theta}$ of various chain lengths; our best fit yields $\tau_{c} \sim \xi^{-0.46 \pm 0.04}$ (Fig. 6(a) shows the case of polymer of $N=160$ beads). We note that there no previous simulations have yielded reliable results for the scaling law between $\tau_{c}$ and $\xi$. Buguin et al. ${ }^{7}$ once proposed $\tau_{c} \sim \xi^{-2}$ by a phenomenological model that neglected the hydrodynamic interactions. Our results are clearly different $-\tau_{c}$ decreases slower with the quench depth than that predicted by them. We emphasize that all the scaling exponents reported in our work are apparent ones. In particular, when adding all the time scales from the three stages to get the total collapse time, the scaling of the latter is expected to be the same as the longest 

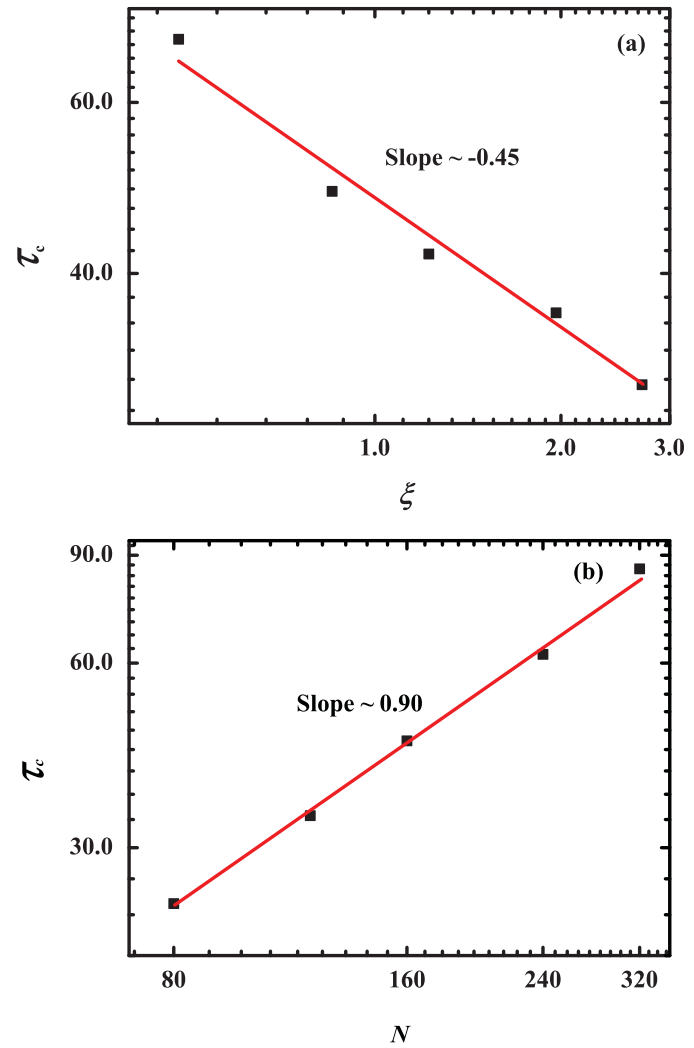

FIG. 6. (a) The log-log plot of the collapse time $\tau_{c}$ vs. quench depth $\xi$ with the same polymer chain length $N=160$ beads. (b) The collapse time $\tau_{c}$ vs. the chain length $N$ at $a_{P S}=60.0 k_{B} T / r_{c}$.

time scale from the three stages. That in some cases we get a scaling exponent in the $N$ dependence for the overall collapse time that is less than some of the stages is a reflection of the error bars in our data. Here we just report the apparent value obtained from numerical data fitting.

For the chain length dependence, the results in Table III show that the collapse time increases with chain length as $\tau_{c} \sim N^{0.98 \pm 0.09}$ (Fig. 6(b) shows the case at $a_{P S}=$ $60.0 k_{B} T / r_{c}$.). We note that some other simulation methods, such as Lee and Kapral, ${ }^{22}$ Pham et al. ${ }^{19}$ also reported linear scaling. Lee and Kapral. ${ }^{22}$ used stochastic rotation dynamics and got $\tau_{c} \sim N$. However, using the same method, Kikuchi et al. ${ }^{10}$ obtained $\tau_{c} \sim N^{4 / 3}$, which agreed well with their own phenomenological model. Pham et al. ${ }^{19}$ used Brownian dynamics also obtained $\tau_{c} \sim N^{1.01}$, but only for shallow quenches when there is no trapping at the sausage-like stage. We do not have a clear understanding of the discrepancy between the various previous studies. However, it is worth noting these aforementioned studies all employed implicit solvent models.

Using the scaling law of the collapse duration $\tau_{c}$ to rescale Figs. 3(a) and 3(b), we find $\left\langle R_{g}^{2}(t)\right\rangle$ with different quench depths $\xi$ and chain lengths $N$ can superpose well with each other as shown in Figs. 7(a) and 7(b).

Finally, we obtain the overall collapse time with different quench depths and different chain lengths as $\tau_{c}$ $\sim \xi^{-0.46 \pm 0.04} N^{0.98 \pm 0.09}$.
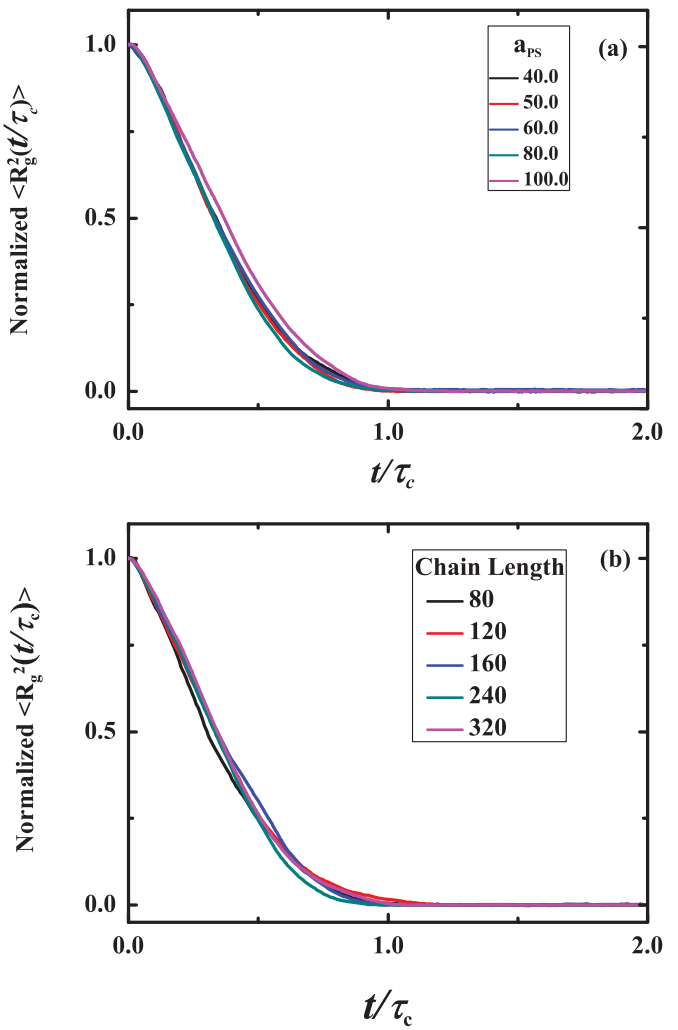

FIG. 7. (a) Normalized $\left\langle R_{g}^{2}(t)\right\rangle$ vs. scaled $t$ at different quench depths. (b) Normalized $\left\langle R_{g}^{2}(t)\right\rangle$ vs. scaled $t$ for different chain lengths.

\section{CONCLUSIONS}

In this work, dissipative particle dynamics simulation is employed to investigate the dynamics of coil-to-globule transition. The focus of this paper is to investigate the collapse pathway and the many-body interaction of solvent particles on the collapse pathway. According to our results, the collapse follows the "pearl-necklace" mechanism with four stages without trapping into a metastable "sausage-like" conformation as seen in implicit solvent methods. The first stage is characterized by formation of localized clusters which in some respects resembles spinodal decomposition, but fails to exhibit some key signatures of spinodal decomposition, such as characteristic length scale corresponding to the fastest growing Fourier mode in the density fluctuation and the growth of this mode. The second to third stages correspond to various coarsening processes with different mechanisms for each stage: coarsening in situ, coarsening involving global backbone motion into a spherical globule. The growth of clusters in size satisfies $\left\langle S_{n}(t)\right\rangle \sim t^{0.95 \pm 0.08}$ at a given quench depth. During the last stage, the crumpled globule relaxes into a compacted globule eventually. By monitoring the normalized solvent molecules in contact with the polymer $\bar{N}_{S}(t)$, the first three stages are identified and the time scale of each stage is obtained. The overall collapse time is shown to scale with the quench depth $\xi$ and the chain length $N$ as $\tau_{c} \sim \xi^{-0.46 \pm 0.04} N^{0.98 \pm 0.09}$. Many of these results differ from those reported in earlier analytical and simulation studies that did not include solvents explicitly. 


\section{ACKNOWLEDGMENTS}

J. Guo gratefully acknowledges the hospitality of the California Institute of Technology, where the work was carried out when she was a visiting student. She is also grateful for the financial support provided by the Program of the National Natural Science Foundation of China (Nos. 20874094, 20934004, and 21004059), NBRPC (No. 2010CB934500), and "Bairen" fund of CAS.

${ }^{1}$ W. H. Stockmayer, Makromol. Chem. 35, 54 (1960).

${ }^{2}$ P. G. de Gennes, Scaling Concepts in Polymer Physics (Cornell University, Ithaca, 1979).

${ }^{3}$ B. Chu, Q. Ying, and A. Grosberg, Macromolecules 28, 180 (1995).

${ }^{4}$ J. Xu, Z. Zhu, S. Luo, C. Wu, and S. Liu, Phys. Rev. Lett. 96, 27802 (2006).

${ }^{5}$ X. Ye, Y. Lu, L. Shen, Y. Ding, S. Liu, G. Zhang, and C. Wu, Macromolecules 40, 4750 (2007).

${ }^{6}$ P. G. de Gennes, J. Phys. (France) Lett. 46, 639 (1985).

${ }^{7}$ A. Buguin, F. Brochard-Wyart, and P. G. de Gennes, C. R. Acad. Sci., Ser. IIb: Mec., Phys., Chim., Astron. 322, 741 (1996).

${ }^{8}$ A. Halperin and P. Goldbart, Phys. Rev. E 61, 565 (2000).

${ }^{9}$ L. Klushin, J. Chem. Phys. 108, 7917 (1998).

${ }^{10}$ N. Kikuchi, J. Ryder, C. Pooley, and J. Yeomans, Phys. Rev. E 71, 61804 (2005).

${ }^{11}$ A. Grosberg, S. Nechaev, and E. Shakhnovich, J. Phys. (Paris) 49, 2095 (1988).

${ }^{12}$ E. Timoshenko and K. Dawson, Phys. Rev. E 51, 492 (1995).

${ }^{13}$ E. Timoshenko, Y. Kuznetsov, and K. Dawson, J. Chem. Phys. 102, 1816 (1995).
${ }^{14}$ Y. Kuznetsov, E. Timoshenko, and K. Dawson, J. Chem. Phys. 104, 3338 (1996).

${ }^{15}$ A. Byrne, P. Kiernan, D. Green, and K. Dawson, J. Chem. Phys. 102, 573 (1995).

${ }^{16}$ Y. Kuznetsov, E. Timoshenko, and K. Dawson, J. Chem. Phys. 103, 4807 (1995).

${ }^{17}$ E. Pitard and H. Orland, EPL 41, 467 (1998).

${ }^{18}$ E. Pitard, Eur. Phys. J. B 7, 665 (1999).

${ }^{19}$ M. Pham, T. Bajaj, and J. Prakash, Soft Matter 4, 1196 (2008).

${ }^{20}$ T. Pham, B. Duenweg, and J. Prakash, Macromolecules 43, 10084 (2010).

${ }^{21}$ N. Kikuchi, A. Gent, and J. Yeomans, Eur. Phys. J. E 9, 63 (2002).

${ }^{22}$ S. H. Lee and R. Kapral, J. Chem. Phys. 124, 214901 (2006).

${ }^{23}$ R. Chang and A. Yethiraj, J. Chem. Phys. 114, 7688 (2001).

${ }^{24}$ J. Polson and M. Zuckermann, J. Chem. Phys. 113, 1283 (2000).

${ }^{25}$ J. Polson and M. Zuckermann, J. Chem. Phys. 116, 7244 (2002).

${ }^{26}$ G. Reddy and A. Yethiraj, Macromolecules 39, 8536 (2006).

${ }^{27}$ K. Kamata, T. Araki, and H. Tanaka, Phys. Rev. Lett. 102, 108303 (2009).

${ }^{28}$ P. Hoogerbrugge and J. Koelman, EPL 19, 155 (1992).

${ }^{29}$ J. Koelman and P. Hoogerbrugge, EPL 21, 363 (1993).

${ }^{30}$ R. D. Groot and P. B. Warren, J. Chem. Phys 107, 4423 (1997).

${ }^{31}$ R. Groot and K. Rabone, Biophys. J. 81, 725 (2001).

${ }^{32}$ A. Alexander-Katz and R. Netz, Macromolecules 41, 3363 (2008).

${ }^{33}$ W. Hu, J. Chem. Phys. 109, 3686 (1998).

${ }^{34}$ C. Wu and X. Wang, Phys. Rev. Lett. 80, 4092 (1998).

${ }^{35}$ J. Cahn, J. Chem. Phys. 42, 93 (1965).

${ }^{36}$ C. Abrams, N. Lee, and S. Obukhov, Europhys. Lett. 59, 391 (2002).

${ }^{37}$ G. E. Crooks, B. Ostrovsky, and Y. Bar-Yam, Phys. Rev. E 60, 4559 (1999).

${ }^{38}$ B. Ostrovsky and Y. Bar-Yam, Europhys. Lett. 25, 409 (1994).

${ }^{39}$ H. Tanaka, J. Phys.: Condens. Matter 17, S2795 (2005). 